\title{
The Effectiveness of Endobronchial Ultrasound-Guided Transbronchial Needle Aspiration in Patients With Undiagnosed Lung Cancer
}

Şule Gül', [MD]

ORCID: 0000-0002-7162-2611

Elif Yelda Niksarlıoğlu', [MD]

ORCID: 0000-0002-6119-6540

Ayşe Yeter ${ }^{1}$

ORCID: 0000-0002-1940-1248

\section{neD ABSTRACT Cen}

Objectives: Conventional bronchoscopic techniques and computed tomography-guided transthoracic needle aspiration are widely used in the diagnosis of lung cancer. In some patients diagnosis can be challenging. Endobronchial ultrasound-guided transbronchial needle aspiration can be used in the diagnosis of lung cancer after procedures have failed to provide a diagnosis. We aimed to show the effectiveness of Endobronchial ultrasound-guided transbronchial needle aspiration in the diagnosis of lung cancer in view of the literature and to share the experience from Turkey.

Material and Methods: This was a retrospective study conducted between 2014 and 2019. Forty-five patients who were suspected of having lung cancer and underwent Endobronchial ultrasound because diagnosis was not confirmed using methods such as bronchoscopy, computed tomography transthoracic needle aspiration, and peripheral lymph node excision, were included in the study.

Results: Three hundred sixty-eight Endobronchial ultrasound procedures were performed. Forty-five patients met the inclusion criteria and were included in the study. Using Endobronchial ultrasound, samples were taken from only mass in eight patients (17.8\%), lymph nodes in 30 patients $(66.7 \%)$, and mass + lymph node in seven (15.5\%) patients. Minor complications were seen in five $(11.1 \%)$ patients and no major complications were seen. Definitive diagnosis was obtained in 35 (77.7\%) patients with Endobronchial ultrasound guided transbronchial needle aspiration. Non-small cell lung cancer was identified in 16 patients $(45.7 \%)$, small cell lung cancer was seen in 15 (42.8\%) patients. Seven of ten undiagnosed patients underwent surgical procedures.

Conclusion: Endobronchial ultrasound, is an effective and safe method for diagnosing lung cancer after undiagnosed procedures. In selected cases, it can be the first choice for the diagnosis of lung cancer.

Keywords: Diagnosing, endobronchial ultrasonography, lung cancer

https://doi.org/10.32552/2021.ActaMedica.557

Received: 20 January 2021, Accepted: 31 March 2021,

Published online: 30 August 2021 


\section{INTRODUCTION}

In patients suspected of having lung cancer, rapid diagnosis and staging are essential for early treatment. Flexible bronchoscopy (FB), computed tomography-guided transthoracic needle aspiration (CT-TTNA) and sputum cytology can be used for diagnosing lung cancer $[1,2]$. Sampling procedures with flexible bronchoscopy such as biopsy, needle aspiration, brush and bronchial lavage have a high diagnostic yield in endobronchial tumors, but the diagnosis rate decreases without endobronchial abnormalities [3]. Transbronchial needle aspiration (TBNA) can increase the diagnostic rate in some extraluminal tumors and can be used in staging, but it is a blind procedure with high false-negative rates; therefore, the yield for TBNA varies widely (14-91\%) [4]. CTTTNA can be used in suitable patients, especially in peripheral masses, but the risk of complications such as pneumothorax and bleeding and using it only in peripheral lesions, restricts its use $[1,5]$.

Endobronchial ultrasound-guided transbronchial needle aspiration (EBUS-TBNA) is widely used in staging lung cancer. The diagnostic efficiency of EBUS in mediastinal staging is around $90 \%$ and at guidelines it was recommended as first option for mediastinal staging before mediastinoscopy [6]. EBUS can also be used for the diagnosis of intrapulmonary tumors, unknown hilar and/or mediastinal lymphadenopathy and pulmonary embolism [7]. In the benign hilar and/or mediastinal lymphadenopathy, the diagnostic accuracy of EBUS warries between $74.5 \%$ - 96\% [8]. EBUS can be used diagnosing lung cancer in patients with no endonronchial lesions. It is effective in diagnosing central peribronchial lung masses and peripheral masses with mediasitinal metastasis. In this group, diagnostic accuracy of EBUS varies 85\%-90\% [1, 9, 10]. However, there are few studies on this subject.

Herein, we aimed to investigate the effectiveness and safety of EBUS in undiagnosed lung cancer despite conventional bronchoscopic techniques and TTNA.

\section{MATERIALS and METHODS}

We designed a retrospective study performed between January 1st, 2014, and November 30th,
2019, in our clinic at an education and research hospital in Turkey. Ethical approval for this study was obtained from the University of Xxx. (8.5.20204/4)

All cases in which EBUS was performed by our clinic during the study period were scanned from the hospital information processing system. Patients with suspected lung cancer and which was not diagnosed with other procedures were included in the study. The inclusion criteria were defined as follows: (1) age over 18 years, (2) suspected lung cancer with thorax $\mathrm{CT}$ and undiagnosed with conventional bronchoscopy, TTNA or peripheral lymph node excision, (3) having extrathoracic malignancy at least 1 year before and suspicion of new primary lung cancer. The exclusion criteria were defined as follows: (1) Suspected cases of lung cancer and that directly underwent EBUS without other previous procedures, (2) cases who were diagnosed with lung cancer and underwent EBUS for mediastinal staging, (3) having active extrathoracic malignancy and suspicion of metastasis.

In all patients, the following data were collected from hospital database: demographic characteristics, comorbidities, CT and positron emission tomography (PET)-CT imaging findings, flexible bronchoscopy and EBUS reports (EBUS duration time, sampling area, number of puncture, number of aspiration, complications) and cytopathologic reports.

\section{Procedure}

Before the procedure, the patients' $\mathrm{CT}$ and PET$\mathrm{CT}$ imaging were examined for the presence of endobronchial lesions and the lesion to be sampled. The procedure was performed using conscious sedation with midazolam and topical lidocaine. Heart rate, blood pressure and oxygen saturation were monitored in real-time. All procedures were performed by a pulmonologist with at least 1 years' EBUS experience. The Convex probe (CP)EBUS scope (Fujifilm EB- 530US with VP-3500HD processor) was used to evaluate the mediastinum, hilum, and parenchymal lung lesion. The target was identified using EBUS and a 22-gauge needle (Echotip*Ultra- ECHO-HD-22-EBUS-O) was advanced using the jabbing technique. Suction 
was used and 12-17 agitations of the needle were performed in the lesion per pass. Samples were sent to cytopathology for analysis. On-site cytology (ROSE) was not used during the bronchoscopic procedures in our hospital. After the procedure, patients were monitored for adverse event detection and registration before being discharged.

\section{Statistical Methods}

Statistical analyses of the study data were performed using the Statistical Package for Social Sciences (SPSS) Version IBM Statistic 21.0 (SPSS Inc., Chicago, IL, USA). Continuous variables were presented as mean and standard deviation, whereas categorical variables were presented as a number and a percentage. Chi-square testing and t-tests were used for categorical and continuous factors, respectively. Statistical significance was considered as $\mathrm{P}<0.05$.

\section{RESULTS}

During the study period, 368 EBUS procedures were performed. Sixty six cases who underwent EBUS for diagnosis lung cancer without other procedures, 40 patiens who had EBUS for mediastinal staging, 34 patients who had extrathoracic malignancy and underwent EBUS for metastasis and 183 patients who underwent EBUS for benign diseases were excluded. 45 patients met the inclusion criteria and were included in the study (Figure 1). Of these patients, $40(88.9 \%)$ were male. The mean age was $63 \pm 7.3$ (range, 40-79) years. The patients' demographics are summarized in Table 1. The CT findings of the patients are summarized in Table 2. In 17 (41.4\%) patients, the mass was located in the right upper lobe and 24 (58.5\%) patients had a mass in the right lobe. Twenty-two (55\%) patients had parenchymal mass and lymphadenopathy; lymphadenopathies were sampled for diagnosis in this group. Eighteen (45\%) patients had mediastinal mass with/without parenchymal mass. Thirty-eight (92.6\%) patients had lymphadenopathy. Among the total 119 lymphadenopathies, were mostly seen in 4R (20.1\%), 7 (20.1\%), and 10R (15.9\%) locations.

Thirty-six (13.3\%) patients had been initially submitted to a non-diagnostic $F B$, six (13.3\%) patients to CT-TTNA, one (2.2\%) to FB+CT-TTNA, and one (2.2\%) patient to peripheral lymphadenopathy excision. In FB, 17 (45.9\%) patients underwent
TBNA, four patients (10.8\%) had bronchial biopsy, and bronchial lavage was performed in all cases (Figure 2). Thirty-one patients (68.9\%) had PET-CT. The PET-CT findings are summarized in Table 2. The mean SUV-max value of mass was $11.3 \pm 11$. PET$C T$ positive lymphadenopathies were mostly seen at $4 R, 10 R$, and 7 locations and the mean maximal SUV-max value was $14.3 \pm 9.1$.

The mean surgical duration of EBUS procedure was 29.8 7.9 (range, 15- 50) minutes and all patients were discharged home after the examination. Samples were taken from only mass in eight (17.8\%) patients, lymph nodes in $30(66.7 \%)$ patients, and mass + lymph node in seven (15.5\%) patients. The lesions were punctured $1.8 \pm 0.6$ (range, 1-3) times and at least 12-17 aspirations were performed in each sampling (Figure 2). A total of 57 lymph nodes were sampled in the 37 patients and the sampled lymph node distribution is shown in Table 3. Complications were seen in five (11.1\%) patients. Among these, hypertension attack occurred in two patients, respiratory distress in two patients, and hemorrhage in one patient. There were no problems in the follow-up after the procedure and hospitalization was not required.

Malignancy was diagnosed in 35 (77.7\%) patients using EBUS.Non-small cell lung cancerwasidentified in 16 cases (45.7\%); lung adenocarcinoma in nine $(56.2 \%)$ patients, squamous cell carcinoma in six

Table 1. Patients' demographics

\begin{tabular}{|c|c|}
\hline $\mathrm{N}=45$ & Number (\%) \\
\hline Age & $63 \pm 7.3$ \\
\hline Male/Female & $\begin{array}{c}40(88.9 \%) / 5 \\
(11.1 \%)\end{array}$ \\
\hline \multicolumn{2}{|l|}{ Smoking $(n=44$} \\
\hline Nonsmoker & $3(7 \%)$ \\
\hline Exsmoker & $10(23 \%)$ \\
\hline Smoker & $31(70 \%)$ \\
\hline P/Year & $40.1 \pm 22.0$ \\
\hline \multicolumn{2}{|l|}{ Comorbidities $(n=44)$} \\
\hline None & $16(\% 36)$ \\
\hline $\mathrm{HT}+\mathrm{IHD} \mathrm{D}^{*}$ & $14(\% 32)$ \\
\hline COPD† & $11(\% 25)$ \\
\hline DMキ & $5(\% 11)$ \\
\hline Previous malignancy & $5(\% 11)$ \\
\hline Depression & $1(\% 2)$ \\
\hline Histiocytosis $\mathrm{x}$ & $1(\% 2)$ \\
\hline
\end{tabular}

(*HT-Hypertension, IHD-Ischemic heart disease, +COPD-Chronic obstructive pulmonary disease, ҒDM-Diabetes mellitus) 


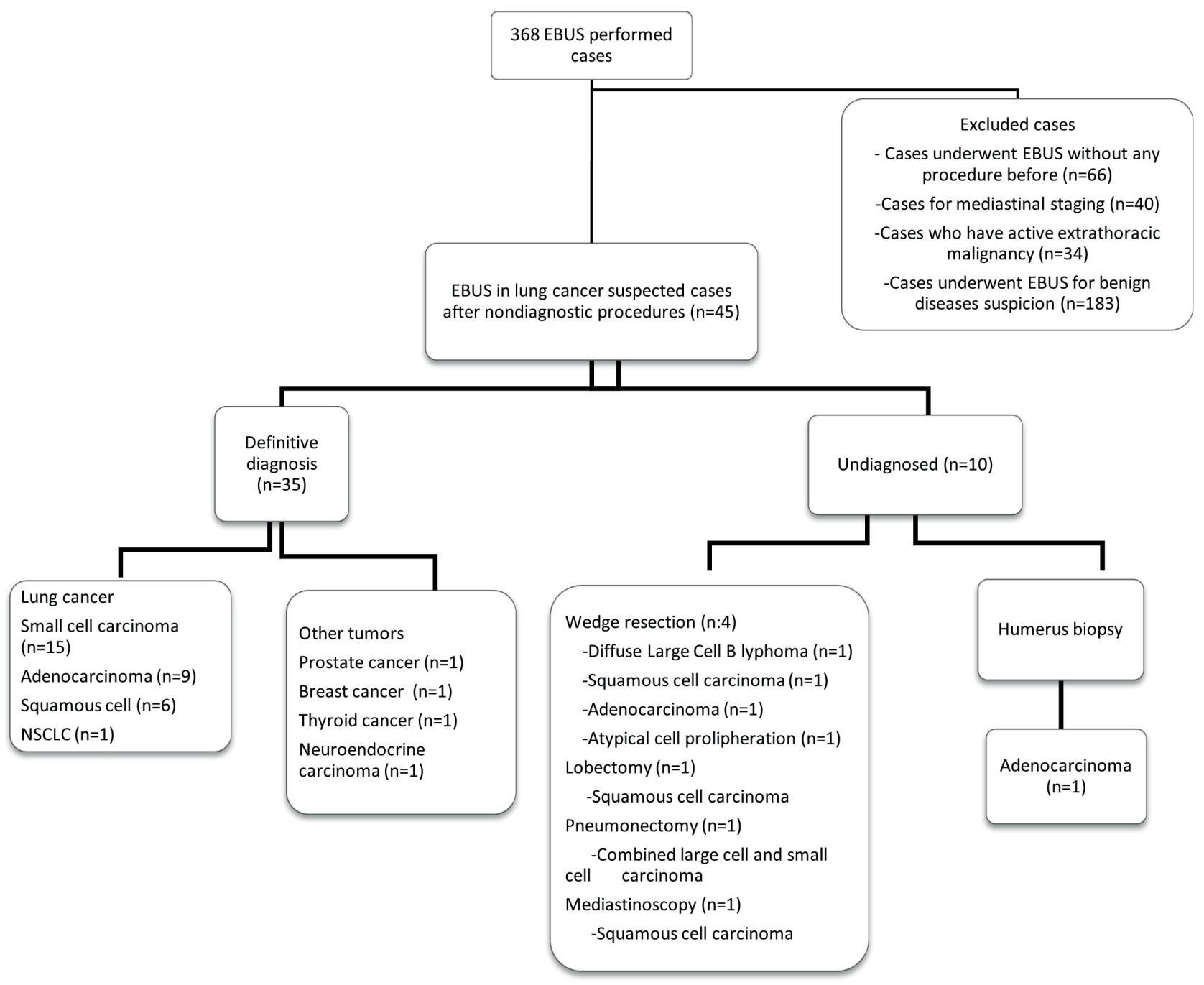

Figure 1. Diagnosis chart of patients underwent EBUS

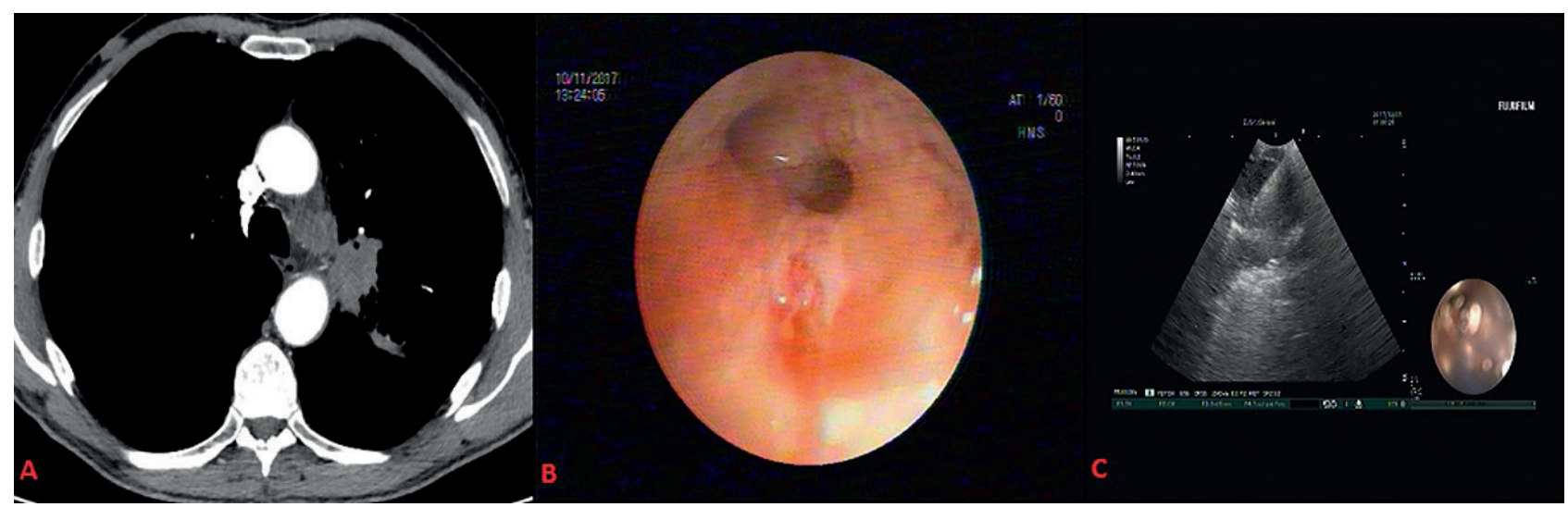

Figure 2. A-Left hilar $32 * 26 \mathrm{~mm}$ mass and $34 * 24 \mathrm{~mm}$ lympadenopathy at $4 \mathrm{~L}$. B- TBNA performed from secondary carina but diagnosis could not be obtained. C- $4 \mathrm{~L}$ and left hilar area was sampled with EBUS. Diagnosis was squamous cell carcinoma.

(37.5\%) patients, and undifferentiated carcinoma in one (6.2\%) patient. Small cell lung cancer was seen in $15(42.8 \%)$ patients. In four patients, prostate cancer, breast cancer, medullary thyroid carcinoma, and high-grade neuroendocrine carcinoma were identified.
In ten undiagnosed patients, seven patients underwent further procedures: wedge resection $(n=4)$, lobectomy $(n=1)$, pneumonectomy $(n=1)$, and mediastinoscopy $(n=1)$. One patient's cancer was diagnosed through a biopsy from the metastatic lesion in the humerus. These patients 
Table 2. Computed tomography and PET-CT findings

\begin{tabular}{|c|c|}
\hline Mass location $(n=41)$ & Number (\%) \\
\hline $\mathrm{RUL}^{*}$ & $17(41.4 \%)$ \\
\hline RML† & $3(7.3 \%)$ \\
\hline RLL‡ & $4(9.7 \%)$ \\
\hline LUL§ & $9(21.9 \%)$ \\
\hline LLL $\|$ & $7(17 \%)$ \\
\hline No mass & $1(2.4 \%)$ \\
\hline Mediastinal mass & $6(15 \%)$ \\
\hline Parenchymal mass & $22(55 \%)$ \\
\hline Mediastinal+parenchymal mass & $12(30 \%)$ \\
\hline Mean size of mass (mm) & $37.9 \pm 25.2$ \\
\hline \multicolumn{2}{|l|}{ Lymphadenopathy } \\
\hline Yes & $38(92.6 \%)$ \\
\hline No & $3(7.3 \%)$ \\
\hline Lymph node locations ( $\mathrm{n}=119)$ & $\begin{array}{c}\text { Number of lymph } \\
\text { nodes }\end{array}$ \\
\hline $2 \mathrm{R}$ & $4(3.3 \%)$ \\
\hline $2 \mathrm{~L}$ & $1(0.8 \%)$ \\
\hline 3 & $3(2.5 \%)$ \\
\hline $4 \mathrm{R}$ & $24(20.1 \%)$ \\
\hline $4 \mathrm{~L}$ & $13(10.9 \%)$ \\
\hline 5 & $2(1.6 \%)$ \\
\hline 6 & $13(10.9 \%)$ \\
\hline 7 & $24(20.1 \%)$ \\
\hline 8 & $1(0.8 \%)$ \\
\hline $10 R$ & $19(15.9 \%)$ \\
\hline $10 \mathrm{~L}$ & 15 (12.6\%) \\
\hline $\begin{array}{l}\text { Mean size of maximal } \\
\text { lymphadenopathy }(\mathrm{mm})\end{array}$ & $22.5 \pm 16.5$ \\
\hline $\begin{array}{l}\text { PET-CT positive lymphadenopathy } \\
\text { locations }\end{array}$ & Number (\%) \\
\hline $2 \mathrm{R}$ & $6(5.6 \%)$ \\
\hline $2 \mathrm{~L}$ & $2(1.8 \%)$ \\
\hline 3 & $3(2.8 \%)$ \\
\hline $4 \mathrm{R}$ & $18(16.9 \%)$ \\
\hline $4 \mathrm{~L}$ & $10(9.4 \%)$ \\
\hline 5 & 4 (3.7\%) \\
\hline 6 & 11 (10.3\%) \\
\hline 7 & 15 (14.1\%) \\
\hline 8 & 4 (3.7\%) \\
\hline $10 \mathrm{R}$ & 17 (16\%) \\
\hline $10 \mathrm{~L}$ & 12 (11.3\%) \\
\hline $11 \mathrm{R}$ & $3(2.8 \%)$ \\
\hline $11 \mathrm{~L}$ & 1 (0.9\%) \\
\hline
\end{tabular}

(*RUL: Right upper lobe, +RML: Right middle lobe, $\neq$ RLL: Right lower lobe, §LUL: Left upper lobe, ||LLL: Left lower lobe)
Table 3. EBUS procedure details

\begin{tabular}{|l|c|}
\hline Mean surgical time & $\begin{array}{c}29.8 \pm 7.9 \\
\text { (range } 15-50 \mathrm{~min})\end{array}$ \\
\hline Total lymph nodes punctured & $1.8 \pm 0.6(1-3)$ \\
\hline Punctured lymph node locations & 57 \\
7 & $20(35 \%)$ \\
$4 \mathrm{R}$ & $16(28 \%)$ \\
$10 \mathrm{~L}$ & $9(15 \%)$ \\
$4 \mathrm{~L}$ & $8(14 \%)$ \\
$10 \mathrm{R}$ & $3(5 \%)$ \\
$11 \mathrm{R}$ & $1(1.7 \%)$ \\
\hline
\end{tabular}

were diagnosed as having squamous cell carcinoma $(n=3)$, adenocarcinoma $(n=2)$, combined large cell and small cell carcinoma $(n=1)$, diffuse large b cell lymphoma $(n=1)$, and atypical cellular proliferation $(n=1)$. Information about the final diagnosis of two patients could not be obtained (Figure 1).

The diagnostic accuracy of EBUS was found as $77.7 \%$. If we separate patients by sampled mediastinal masses and sampled lymph nodes, mediastinal masses were sampled using EBUS in 15 (33.3\%) patients and 14/15 (93.3\%) patients received a diagnosis. Lymph nodes were sampled in 37 patients (82.2\%) and the diagnostic accuracy was $75.6 \%(28 / 37)$ in this group. There was no relation between diagnosis with EBUS and age, mean lymph node size, mean mass size, SUV-max of mass and lymph node at PET-CT, and the number of samples per lesion. The mean surgical time was correlated with the diagnostic rate, which was 31.6 min in cases diagnosed with EBUS and $26.5 \mathrm{~min}$ in undiagnosed cases $(p=0.028)$ (Table 4). There are differences in the number of patients between the subgroups due to the absence of lymph nodes or PET-CT in each patient.

\section{DISCUSSION}

In our study, a $77.7 \%$ diagnostic yield was shown in patients with undiagnosed lung cancer with acceptable and no serious complications. Diagnostic success increases to $93.3 \%$ in patients sampled the mass. Duration time of EBUS was correlated with diagnosis and there is no data on this subject in previous studies. 
Table 4. Factors that may be associated with efficiacy of EBUS

\begin{tabular}{|l|c|c|c|}
\hline \multirow{2}{*}{ Age } & \multicolumn{2}{|c|}{ EBUS sampling } & \multirow{2}{*}{ p value } \\
\cline { 2 - 4 } Mean size of mass $(\mathrm{mm})^{*}$ & Diagnostic $(\mathrm{n}=35)$ & Nondiagnostic $(\mathrm{n}=10)$ & 0.586 \\
Mean size of maximal lymphadenopathy $(\mathrm{mm}) \dagger$ & $62.9 \pm 7.6$ & $64.3 \pm 6.3$ & 0.362 \\
Mean SUV-max value of massł & $40.9 \pm 21.5$ & $48.78 \pm 26.1$ & 0.922 \\
Mean maximal SUV-max value of & $27.54 \pm 11$ & $27 \pm 21.5$ & 0.984 \\
lymphadenopathy§ & $16.9 \pm 9$ & $16.9 \pm 10.7$ & 0.845 \\
Mean surgical duration of EBUS procedure $(\mathrm{min})$ & $15.45 \pm 8.2$ & $14.7 \pm 10.3$ & $\mathbf{0 . 0 2 8}$ \\
\hline Number of samples per lesion & $31.6 \pm 6$ & $26.5 \pm 7.1$ & 0.583 \\
\hline
\end{tabular}

* 31 patients in EBUS diagnostic group and 9 patients in EBUS nondiagnostic group

+28 patients in EBUS diagnostic group and 9 patients in EBUS nondiagnostic group

$\neq 23$ patients in EBUS diagnostic group and 7 patients in EBUS nondiagnostic group

$\S 22$ patients in EBUS diagnostic group and 6 patients in EBUS nondiagnostic group

EBUS has been widely used for staging lung cancer and has been included in guidelines for mediastinal staging [11]. Gu et al. reported the sensitivity rate of EBUS in staging as 93\% [12], and a recently published study by Guarize et al. showed a sensitivity of $90.7 \%$, and diagnostic accuracy of $93.1 \%$ in staging lung cancer [9]. Dhooria et al. showed that a combination of EUS ad EBUS increased the diagnostic rate $(80 \%$ to $91 \%)$ in the staging of mediastinal lung cancer [13].

Lung cancer diagnosis is challenging in some patients. Although FB and TTNA are the first procedures for diagnosing lung cancer, it was shown that $12 \%$ of cases were undiagnosed after these procedures [1]. In a review, the diagnostic accuracy of different modalities using by $F B$ was shown as $74 \%$ with endobronchial biopsy, $59 \%$ with cytobrush, and $48 \%$ with bronchial lavage in endobronchial lesions in patients with suspected lung cancer. The combined sensitivity for all modalities was $88 \%$. It was observed that sensitivity decreased in peripheral lesions [14]. TBNA can increase the diagnostic rate, but it also has limitations. It was shown in a retrospective study that although the diagnostic accuracy of lymph nodes $\geq 2 \mathrm{~cm}$ was $93 \%$, diagnostic accuracy decreased to $89.7 \%$ in lymph nodes $<2 \mathrm{~cm}$ because of blind visualization [15].

CT-TTNA is an effective method for the diagnosis of lung cancer, especially in peripheral nodules and masses. In different studies, its diagnostic accuracy was found ranging from $64 \%$ to $97 \%$ because its efficiency depends on many factor such as the size and location of the lesion, biopsy technique, needle type, number of passes, and operator experience. Also, there are limitations such as adequate pulmonary function tests, and no bleeding tendency. It is a method that can be used in selected patients due to its adverse effects such as pneumothorax and bleeding [16].

Before EBUS, the patients who had undiagnosed lung cancer with conventional bronchoscopic techniques and TTNA, underwent surgical procedures such as mediastinoscopy and thoracoscopy. However with standard cervical mediastinoscopy, paratracheal (station 2 and 4) and subcarinal (station 7) lymph nodes are available. In the study by Ernst et al. when the diagnostic success of EBUS and mediastinoscopy was compared, it was found that the diagnostic success of EBUS was superior with a rate of $91 \%$ to $78 \%$. While no complications related to EBUS were observed, complications such as wound infection and bleeding were observed after mediastinoscopy. In different studies, mediastinoscopy has a higher complication rate with mortality reported between 0.08 and $0.2 \%$ and a morbidity rate of $2 \%$ and $2.5 \%$. Repeatability is another advantage of EBUS [17].

While using for lung cancer staging, EBUS was found to be useful for diagnosing lung cancer, especially in central masses with no endobronchial lesions and metastatic central lymph nodes with peripheral lesions. There are some studies showing the effectiveness of EBUS after nondiagnostic procedures such as FB and CT-TTNA. Eckardt et al. were able to diagnose $55 \%$ of 308 patients with 
EBUS after nondiagnostic procedures [18]. In 163 patients who had centrally located intrapulmonary tumors with no endobronchial abnormalities, EBUS detected tumor in 145/163 (89\%) and a definitive diagnosis was achieved in $94 \%$ of patients $(136 / 145)$ in another study [3]. Tournoy et al. reported a sensitivity of $84 \%$ in the diagnosis of central lung lesions not visible in routine bronchoscopy [19]. In a study including the largest EBUS series, EBUSTBNA demonstrated a sensitivity of $90.9 \%$ and accuracy as $91.7 \%$ in paratracheal or peribronchial pulmonary lesions [9]. In our study, similar to the studies \%93,3 of patients who have mediastinal masses diagnosed with EBUS. Mass size, mass SUVmax value in PET-CT, and sampling number were not correlated with EBUS diagnostic yield. With these higher diagnostic rates, EBUS can be the first choice in diagnosing lung cancer in mediastinal tumors without endobronchial lesions.

In metastatic central lymph nodes with parenchymal masses, EBUS is also an effective tool for diagnosis. In a study by Conte et al., EBUS was shown to be effective in lymph nodes under $2 \mathrm{~cm}$ with $94.2 \%$ diagnostic accuracy and $93 \%$ sensitivity [15]. Bugalho et al. used EBUS and EUS together and achieved a definitive diagnosis with $89.8 \%$ sensitivity and $100 \%$ specificity after nondiagnostic FB and TTNA. They stated that applying two procedures together increased the rate of diagnosis [1]. In a review article, Colella et al. reported that the sensitivity of EBUS ranged from $85 \%$ to $97 \%$ [20]. In our study, in 37 patients (82.2\%) lymph nodes were sampled and diagnostic accuracy was $75.6 \%$ in this group. There was no relation between diagnostic accuracy and lymph node size, sampling number and lymph node SUV-max value in PETCT. In different studies there was no statistically significant relationship between lymph node size and EBUS diagnostic success as in our study [15, 21]. In Marchand's et al. study, EBUS sensitivity rate was 33\% in low PET-CT activity (SUV $<4)$ and $\% 79$ in high PET-CT activity (SUV>4) [21]. The reason that we could not find a relationship between PET involvement and lymph node diagnosis success in our study; may be the high PET involvement in almost all our patients and sampling of lymph node with high PET involvement.

The diagnostic effectiveness of EBUS may also depend on the number of aspirations, although there is no consensus on this issue yet. If rapid on- site evaluation (ROSE) is available, the number is not very important, but unfortunately, ROSE cannot be used in many centers, like our center. In several studies, it was demonstrated that an optimal result could be obtained after the third EBUS-TBNA pass per lesion $[22,23]$. However, in other studies, there was no significant correlation between diagnostic accuracy and the number of passes [24, 25]. In our study, we sampled each lesion (lymph node or mass) $1.8 \pm 0.6$ (1-3) times and at least 12-17 aspirations were performed with sampling.

Unlike other studies, our data showed that surgical time was associated with the diagnostic efficacy of EBUS. The surgical duration was 31.6 minutes in cases diagnosed with EBUS, and 21.5 minutes in undiagnosed cases and the difference was statistically significant. In a study by Bugalho et al., the mean surgical time was 35.5 minutes. However, in this study and other studies, it was not stated whether surgical time was correlated with the diagnosis $[1,3,10]$.

The optimal method for sedation, a factor that may affect the diagnostic accuracy of EBUS, is still controversial. In a review including six studies, there was no specific difference in diagnostic efficacy and complication rates between the deep sedation and moderate sedation groups [26]. Some researchers suggest that using deep sedation especially sampling EBUS-visible intrapulmonary lesions [27]. We use moderate sedation with midazolam and topical lidocaine in our institution. Considering the patients who developed complications, the operation could not be continued related to high blood pressure in only one patient. Therefore, we think that the place of sedation in the success of the procedure may be limited.

No major complications were seen in our study. In five (11.1\%) patients, minor complications were seen but neither needed hospitalization. Although there were no major complications in many studies, minor complication rates range from $0-12.5 \%[1,3$, 28]. These minor complications may occur because of moderate sedation.

In Navani et al.'s study, in patients with non-small cell lung cancer diagnosed using EBUS, survival was longer compared with patients diagnosed with conventional methods (503 vs. 312 days). In subgroup analysis, patients with lung cancer 
who underwent surgery had better postoperative survival in the EBUS group. The authors stated that sampling mediastinal lymph nodes that anatomically drained the primary tumor might result in improved survival in the patient group undergoing surgery. Early treatment decision and early treatment could also improve survival [6]. Further studies are needed on the survival effect of EBUS.

The retrospective nature of this study is the major limitation. Therefore, randomization of patients could not be achieved and the indication for the procedure was determined by different physicians. This caused deficiencies in data collection. Another limitation is the small number of cases. Also, this study was conducted in a single-center. Future, multi-centered, randomized studies may guide EBUS's diagnostic effectiveness as well as costeffectiveness and survival contribution.

\section{CONCLUSION}

EBUS is an effective and safe method in the diagnosis of lung cancer that is undiagnosed with other procedures. EBUS may be the first diagnostic procedure for mediastinal and peripheral masses, especially with mediastinal lymphadenopathies. Detailed examination of CT findings and appropriate patient selection may increase diagnosis rates. Reduction in the time-to-treatment decision might improve survival in patients with lung cancer. Further investigations are needed to determine the survival effectiveness of EBUS.

\section{CONFLICT Of INTEREST}

All authors stated that there is not any conflict of interest.
[1] Bugalho A, Ferreira D, Eberhardt R, et al. Diagnostic value of endobronchial and endoscopic ultrasound-guided fine needle aspiration for accessible lung cancer lesions after non-diagnostic conventional techniques: A prospective study. BMC Cancer; 2013; 19;13:130.

[2] Su J, Anjuman N, Guarnera MA, et al. Analysis of Lung Flutecollected Sputum for Lung Cancer Diagnosis. Biomarker Insights 2015; 10: 55-61.

[3] Kuijvenhoven JC, Livi V, Morandi L, et al. The expanding role of endobronchial ultrasound in patients with centrally located intrapulmonary tumors. Lung Cancer 2019; 134: 194-201.

[4] Yasufuku K, Fujisawa T. Staging and diagnosis of non-small cell lung cancer: Invasive modalities. Respirology 2007; 12: 173-183.

[5] Herth F, Becker HD, Ernst A. Conventional vs Endobronchial Ultrasound-Guided Transbronchial Needle Aspiration. Chest 2004; 125: 322-325.

[6] Navani N, Nankivell M, Lawrence DR, et al. Lung cancer diagnosis and staging with endobronchial ultrasoundguided transbronchial needle aspiration compared with conventional approaches: An open-label, pragmatic, randomised controlled trial. The Lancet Respiratory Medicine 2015; 3: 282-289.

[7] Li P, Zheng W, Zhao L. Convex probe endobronchial ultrasound: Applications beyond conventional indications. Journal of Thoracic Disease 2015; 7: E289-E297.

[8] Shen H, Lou L, Chen T, et al. Comparison of transbronchial needle aspiration with and without ultrasound guidance for diagnosing benign lymph node adenopathy. Diagnostic Pathology 2020; 15: 1-9.
[9] Guarize J, Casiraghi M, Donghi S, et al. Endobronchial Ultrasound Transbronchial Needle Aspiration in Thoracic Diseases: Much More than Mediastinal Staging. Canadian Respiratory Journal; 2018:4269798

[10] Argento AC, Puchalski J. Convex probe EBUS for centrally located parenchymal lesions without a bronchus sign. Respiratory Medicine 2016; 116: 55-58.

[11] Leyn D, P. D leyn, C. D, et al. Revised ests guidelines for preoperative mediastinal lymph node staging for nonsmall-cell lung cancer. European Journal of Cardiothoracic Surgery 2014; 3: 225-233.

[12] Gu P, Zhao YZ, Jiang LY, et al. Endobronchial ultrasoundguided transbronchial needle aspiration for staging of lung cancer: A systematic review and meta-analysis. European Journal of Cancer 2009; 45(8):1389-1396.

[13] Dhooria S, Aggarwal AN, Gupta D, et al. Utility and safety of endoscopic ultrasound with bronchoscope- guided fineneedle aspiration in mediastinal lymph node sampling: Systematic review and meta-analysis. Respiratory Care 2015; 60: 1040-1050.

[14] Schreiber G, McCrory DC. Performance characteristics of different modalities for diagnosis of suspected lung cancer: Summary of published evidence. Chest. 2003 Jan;123(1 Suppl):115S-128S.

[15] Conte SC, Spagnol G, Biolo M, et al. A retrospective study of endobronchial ultrasound transbronchial needle aspiration versus conventional transbronchial needle aspiration in diagnosis/staging of hilar/mediastinal lymph node in lung cancer: Which role in clinical practice? Monaldi Archives for Chest Disease 2019; 89: 1-5. 
[16] Manhire A, Charig M, Clelland C, et al. Guidelines for radiologically guided lung biopsy. Thorax 2003; 58: $920-$ 936.

[17] Ernst A, Anantham D, Eberhardt R, et al. Diagnosis of mediastinal adenopathy-real-time endobronchial ultrasound guided needle aspiration versus mediastinoscopy. Journal of Thoracic Oncology 2008; 3: 577-582.

[18] Eckardt J, Olsen KE, Licht PB. Endobronchial ultrasoundguided transbronchial needle aspiration of undiagnosed chest tumors. World Journal of Surgery. 2010; 34(8):18231827.

[19] Tournoy KG, Rintoul RC, van Meerbeeck JP, et al. EBUSTBNA for the diagnosis of central parenchymal lung lesions not visible at routine bronchoscopy. Lung Cancer. 2009; 63(1):45-49.

[20] Colella S, Vilmann P, Konge L, et al. Endoscopic ultrasound in the diagnosis and staging of lung cancer. Endoscopic Ultrasound 2014; 205-212.

[21] Marchand C, Medford ARL. Relationship between endobronchial ultrasound-guided (EBUS)-transbronchial needle aspiration utility and computed tomography staging, node size at EBUS, and positron emission tomography scan node standard uptake values: A retrospective analysis. Thoracic Cancer 2017; 8: 285-290.

[22] Lee HS, Lee GK, Lee HS, et al. Real-time endobronchial ultrasound-guided transbronchial needle aspiration in mediastinal staging of non-small cell lung cancer: How many aspirations per target lymph node station? Chest. 2008; 134(2):368-374.
[23] Ece D, Keser SH, Çağlayan B, et al. Endobronchial ultrasound-guided transbronchial fine needle aspiration: Determinants of adequacy. Turkish Journal of Thoracic and Cardiovascular Surgery 2018; 26: 123-131.

[24] Ye T, Hu H, Luo $\mathrm{X}$, et al. The role of Endobronchial ultrasound guided transbronchial needle aspiration (EBUS-TBNA) for qualitative diagnosis of mediastinal and hilar lymphadenopathy: A prospective analysis. BMC Cancer 2011; 11: 100.

[25] Cetinkaya E, Gunluoglu G, Ozgul A, et al. Value of real-time endobronchial ultrasound-guided transbronchial needle aspiration. Annals of Thoracic Medicine 2011; 6: 77-81.

[26] Aswanetmanee $P$, Limsuwat $C$, Kabach $M$, et al. The role of sedation in endobronchial ultrasound-guided transbronchial needle aspiration: Systematic review. Endoscopic Ultrasound 2016; 5:300-306.

[27] Almeida FA, Salam S, Mehta AC, et al. Sampling Utility of the Convex Probe Endobronchial Ultrasound Visible Intrapulmonary Lesion. Journal of Bronchology and Interventional Pulmonology 2018; 25: 290-299.

[28] Choi YR, An JY, Kim MK, et al. The diagnostic efficacy and safety of endobronchial ultrasound-guided transbronchial needle aspiration as an initial diagnostic tool. Korean Journal of Internal Medicine 2013; 28: 660-667. 